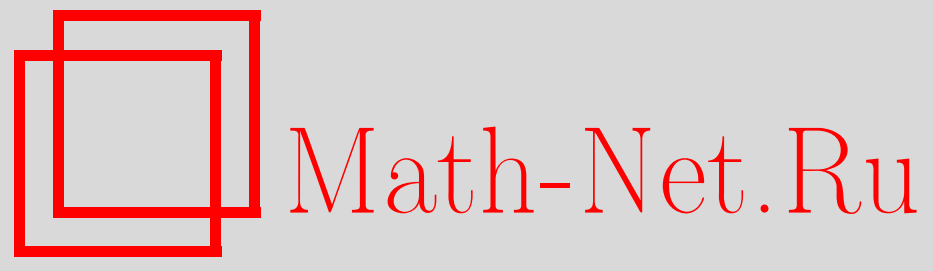

А. М. Локощенко, В. В. Терауд, Е. А. Шеварова, Установившаяся ползучесть длинной мембраны внутри жесткой матрицы при переменном поперечном давлении, Вестн. Сам. гос. техн. ун-та. Сер. Физ.-мат. науки, 2020, номер 3, 445-468

DOI: https://doi.org/10.14498/vsgtu1772

Использование Общероссийского математического портала MathNet.Ru подразумевает, что вы прочитали и согласны с пользовательским соглашением

http: //www.mathnet.ru/rus/agreement

Параметры загрузки:

IP: 54.237 .206 .68

26 апреля 2023 г., 11:54:27

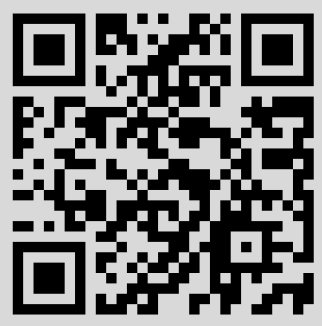


УДК 539.376

\title{
Установившаяся ползучесть длинной мембраны внутри жесткой матрицы при переменном поперечном давлении
}

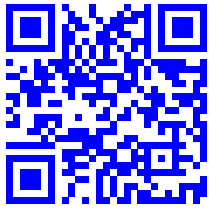

\author{
(C) А. М. Локощенко ${ }^{1}$ В. В. Терауд ${ }^{1,2}$, E. А. Шеварова ${ }^{1,3}$ \\ 1 Московский государственный университет имени М. В. Ломоносова, \\ Научно-исследовательский институт механики, \\ Россия, 119192, Москва, Мичуринский проспект, 1. \\ 2 Самарский государственный технический университет, \\ Россия, 443100, Самара, ул. Молодогвардейская, 244. \\ 3 Московский авиационный институт (национальный исследовательский университет), \\ Россия, 125993, Москва, Волоколамское шоссе, 4.
}

\section{Аннотация}

Исследуется задача об установившейся ползучести длинной прямоугольной мембраны в стесненных условиях внутри жесткой матрицы при кусочно-постоянной зависимости величины поперечного давления $q$ от времени $t$. В задаче рассматривается длинная матрица прямоугольного сечения, в которой отношение ее высоты к ширине не меньше 0.5. В качестве примера исследуется ползучесть мембраны при однократном изменении величины поперечного давления во времени. Рассматриваются три варианта условий контакта мембраны и матрицы: идеальное скольжение, прилипание и скольжение с учетом трения. В данной работе исследованы четыре стадии деформирования мембраны. На первой стадии (упругое деформирование) мембрана, плоская в начальном состоянии, под действием давления $q$ мгновенно упруго деформируется, приобретая форму незамкнутой круговой цилиндрической оболочки с центральным углом $2 \alpha_{1}$. На второй стадии мембрана деформируется в условиях установившейся ползучести вплоть до момента касания боковых стенок матрицы. Третья стадия заканчивается в момент касания мембраной поперечной стенки матрицы. На четвертой стадии мембрана контактирует с матрицей по поперечной и боковым сторонам. Анализ проводится до времени практически полного прилегания мембраны к матрице, при котором отношение радиуса мембраны вблизи углов

\section{Научная статья}

(2) (1) Контент публикуется на условиях лицензии Creative Commons Attribution 4.0 International (https://creativecommons.org/licenses/by/4.0/deed.ru)

\section{Образец для цитирования}

Локощенко А. М., Терауд В. В., Шеварова Е. А. Установившаяся ползучесть длинной мембраны внутри жесткой матрицы при переменном поперечном давлении // Вестн. Сам. гос. техн. ун-та. Сер. Физ.-мат. науки, 2020. Т. 24, № 3. С. 445-468. https://doi.org/10.14498/vsgtu1772.

\section{Сведения об авторах}

Александр Михайлович Локощенко (1) https://orcid.org/0000-0002-5462-6055 доктор физико-математических наук, профессор; заведующий лабораторией; лаб. ползучести и длительной прочности ${ }^{1}$; e-mail: loko@imec.msu.ru

Валентин Викторович Терауд (1) https://orcid.org/0000-0001-8336-5598

канд. техн. наук; старший научный сотрудник; лаб. ползучести и длительной прочности ${ }^{1}$; научный сотрудник; каф. прикладной математики и информатики ${ }^{2}$;

e-mail: Idrnww@gmail .com

Екатерина Алексеевна Шеварова; ведущий инженер; лаб. ползучести и длительной прочности $^{1}$; студент ${ }^{3}$; e-mail: kat121193@уа.ru 
матрицы к начальной ширине мембраны составляет 0.005. Для третьей и четвертой стадий дополнительно учитывается сила трения мембраны о стенки матрицы. Получены зависимости толщины различных частей мембраны от времени, а также интенсивности напряжений в мембране от времени. Применительно к данной постановке задачи рассмотрены отклонения от правила суммирования парциальных времен заполнения матрицы.

Ключевые слова: мембрана, установившаяся ползучесть, матрица, поперечное давление, идеальное скольжение, прилипание, нестационарное нагружение, трение.

Получение: 11 февраля 2020 г. / Исправление: 10 июля 2020 г. / Принятие: 14 сентября 2020 г. / Публикация онлайн: 30 сентября 2020 г.

\section{Введение}

Рассмотрим деформирование длинной узкой прямоугольной мембраны шириной $2 a$ и начальной толщиной $H_{0}$, закрепленной вдоль длинных сторон и нагруженной равномерным поперечным давлением $q$ (рис. 1), которое может изменяться во времени $t$ по заданному закону. Решение этой задачи при различных физических и геометрических условиях приведено в монографиях Одквиста (F. К. G. Odqvist) [1], Л. М. Качанова [2], Н. Н. Малинина [3] и др. Особый интерес представляет исследование ползучести рассматриваемой мембраны внутри жесткой матрицы. В монографиях [3,4] рассмотрен цикл задач о ползучести такой мембраны внутри жесткой матрицы. В [4] приведены решения задач при учете различных форм матриц: клиновидной [5], криволинейной [6] и прямоугольной при различных условиях на контакте мембраны и матрицы. Во всех приведенных решениях величина равномерного поперечного давления $q$ не зависит от времени $t$. В различных решениях использовались разные модели ползучести: установившаяся, неустановившаяся, дробно-степенная. В случае применения дробно-степенной модели ползучести [7] в зависимости от контактных условий с течением времени мембрана либо заполняет пространство внутри матрицы за конечное или бесконечное время, либо разрушается внутри матрицы [5]. В [8] приведено решение аналогичной задачи об установившейся ползучести мембраны при кусочно-постоянной зависимости скорости изменения величины поперечного давления от времени.

В данной работе исследуется ползучесть мембраны внутри матрицы прямоугольной формы. Здесь рассматривается не постоянная величина $q(t)=$ $=$ const, а кусочно-постоянная зависимость величины $q(t)$ с однократным ее изменением. Представляет интерес рассмотрение особенностей практически полного прилегания мембраны к пространству внутри матрицы при скачкообразном увеличении или уменьшении величины $q$.

Для исключения появления в начальный момент времени бесконечных напряжений в данном решении учитывается мгновенное упругое деформирование. 
Для описания деформирования мембраны при $t>0(t$-время) используется степенная модель установившейся ползучести материала

$$
\frac{d p_{u}}{d t}=\frac{1}{t_{0}}\left(\frac{\sigma_{u}}{\sigma_{0}}\right)^{n},
$$

в которой $\sigma_{u}$ и $\dot{p}_{u}-$ интенсивности напряжений и скоростей деформаций ползучести соответственно, $\sigma_{0}, t_{0}$ и $n$ - постоянные величины соответствующей размерности.

Исследование свободного деформирования проводится в двух последовательных стадиях. На первой стадии (упругое деформирование) мембрана, плоская в начальном состоянии, под действием давления $q$ мгновенно упруго деформируется, приобретая форму незамкнутой круговой цилиндрической оболочки с центральным углом $2 \alpha_{1}$. На второй стадии мембрана деформируется в условиях установившейся ползучести вплоть до касания стенок жесткой матрицы, при этом угол раствора мембраны совпадает с углом раствора матрицы и равен $2 \alpha_{2}=\pi$. При моделировании напряженно-деформированного состояния мембраны рассматриваются радиальное $\sigma_{r r}$, окружное $\sigma_{\theta \theta}$ и осевое $\sigma_{z z}$ главные напряжения и соответствующие компоненты тензора деформаций $\varepsilon_{r r}, \varepsilon_{\theta \theta}$ и $\varepsilon_{z z}$, при $t>0$ учитываются только компоненты тензора деформаций ползучести $p_{r r}, p_{\theta \theta}$ и $p_{z z}$.

Рассматривая элемент мембраны, принимая напряжения в элементе равномерно распределенными по толщине и записывая уравнения равновесия в проекциях на нормаль и касательную, получаем

$$
\sigma_{\theta \theta}=\frac{q \rho}{H}, \quad d\left(\sigma_{\theta \theta} H\right)=0
$$

где $\sigma_{\theta \theta}$ - окружное напряжение, $\rho$ - радиус кривизны срединной поверхности, $H$ - толщина мембраны.

Следовательно,

$$
\sigma_{\theta \theta} H=\text { const. }
$$

Сопоставляя (2) и (3), заключаем, что в случае равномерного давления $(q=$ const) радиус кривизны срединной поверхности во всех ее точках один

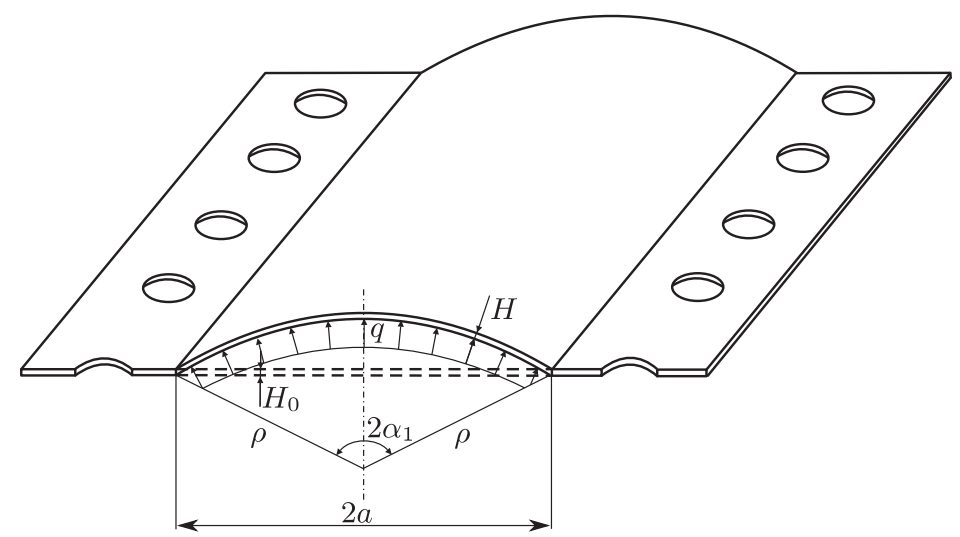

Рис. 1. Геометрическая схема прямоугольной мембраны [Figure 1. Geometry of a rectangular membrane] 
и тот же ( $\rho=$ const), т.е. срединная поверхность мембраны при ее деформировании является частью поверхности кругового цилиндра с некоторым углом раствора $2 \alpha$ [3]. В этом случае очевидно, что если толщина мембраны до деформации постоянна, то она постоянна и после деформации. Следовательно, согласно (2) окружное напряжение по длине окружности радиуса $\rho$ не изменяется.

\section{1. Мгновенное упругое деформирование мембраны (первая стадия)}

Упругое деформирование мембраны описывается с помощью закона Гука при сложном напряженном состоянии с учетом несжимаемости материала. При этом будут использоваться следующие обозначения: $E-$ модуль Юнга, $H$ и $\rho$ - толщина и радиус кривизны поперечного сечения мембраны, $\sigma_{i j}-$ компоненты тензора напряжений. Значения всех рассматриваемых в этом параграфе параметров, полученные в результате приложения поперечного давления $q$, принимают дополнительный индекс 1.

Из условия несжимаемости материала получим значение толщины $H_{1}\left(\alpha_{1}\right)$ :

$$
H_{0} \rho_{1} \sin \alpha_{1}=H_{1} \rho_{1} \alpha_{1}, \quad \frac{H_{1}}{H_{0}}=\frac{\sin \alpha_{1}}{\alpha_{1}} .
$$

Из уравнений равновесия свободной мембраны получим значение напряжения $\sigma_{\theta \theta 1}$ :

$$
q a=H_{1} \sigma_{\theta \theta 1} \sin \alpha_{1}, \quad \sigma_{\theta \theta 1}=\frac{q a}{H_{1} \sin \alpha_{1}} .
$$

Из определения малой деформации получим выражение для окружной деформации $\varepsilon_{\theta \theta 1}$ :

$$
\varepsilon_{\theta \theta 1}=\frac{\rho_{1} \alpha_{1}-a}{a}=\frac{\alpha_{1}}{\sin \alpha_{1}}-1
$$

где $\rho_{1}=a / \sin \alpha_{1}$.

В соответствии с условием несжимаемости используем значение коэффициента Пуассона, равное 0.5. Считаем, что мембрана под поперечным давлением находится в условиях плоского деформированного состояния:

$$
\varepsilon_{z z 1}=0
$$

Для тонкостенных цилиндрических оболочек обычно принимается условие безмоментного напряженного состояния, при этом выполняется равенство $\sigma_{r r 1}=0$.

Из закона Гука при сложном напряженном состоянии следует связь компонент тензоров напряжений $\sigma_{i j}$ и упругих деформаций $\varepsilon_{i j}$ :

$$
\begin{aligned}
& \varepsilon_{z z 1}=E^{-1}\left(\sigma_{z z 1}-0.5 \sigma_{\theta \theta 1}\right), \\
& \varepsilon_{\theta \theta 1}=E^{-1}\left(\sigma_{\theta \theta 1}-0.5 \sigma_{z z 1}\right) .
\end{aligned}
$$

Из равенства (7) при учете (6) получаем

$$
\sigma_{z z 1}=0.5 \sigma_{\theta \theta 1}
$$


Подставляя (9) в (8) и учитывая (2) и (4), получаем:

$$
\varepsilon_{\theta \theta 1}=\frac{3}{4} \frac{\sigma_{\theta \theta 1}}{E}=\frac{3}{4} \frac{q}{E} \frac{\rho_{1}}{H_{1}}=\frac{3}{4} \frac{q}{E} \frac{a}{\sin \alpha_{1}} \frac{\alpha_{1}}{H_{0} \sin \alpha_{1}}=\frac{3 q a \alpha_{1}}{4 E H_{0} \sin ^{2} \alpha_{1}} .
$$

Приравнивая это выражение выражению (5), получаем связь давления $q$ и угла $\alpha_{1}$ :

$$
\frac{3 q a \alpha_{1}}{4 E H_{0} \sin ^{2} \alpha_{1}}=\frac{\alpha_{1}}{\sin \alpha_{1}}\left(1-\frac{\sin \alpha_{1}}{\alpha_{1}}\right), \quad \frac{q}{\sigma_{0}}=\frac{4 H_{0} k}{3 a}\left(1-\frac{\sin \alpha_{1}}{\alpha_{1}}\right) \sin \alpha_{1},
$$

где $k=E / \sigma_{0}$.

Так как упругое деформирование по определению происходит практически мгновенно, длительность первой стадии принимаем равной нулю: $t_{1}=0$.

\section{2. Свободное деформирование мембраны в условиях ползучести (вторая стадия)}

Введем безразмерные переменные:

$$
\begin{gathered}
\bar{q}=\frac{q}{\sigma_{0}}, \quad \bar{H}=\frac{H}{H_{0}}, \quad \bar{H}_{0}=\frac{H_{0}}{a} \\
\bar{t}=\left(\frac{\sqrt{3}}{2}\right) \frac{t}{t_{0}}, \quad \bar{\rho}=\frac{\rho}{a}, \quad \bar{\sigma}_{i j}=\frac{\sigma_{i j}}{\sigma_{0}}, \quad i, j=1,2,3 .
\end{gathered}
$$

Далее черточки над всеми безразмерными переменными опустим. При этом под скоростями всюду понимаются производные по безразмерному времени.

В качестве связи компонент тензоров напряжений и скоростей деформаций ползучести примем гипотезу пропорциональности соответствующих девиаторов $s_{i j}$ (см., например, [4]):

$$
\begin{gathered}
\dot{p}_{i j}=\frac{3 f\left(\sigma_{u}\right)}{2 \sigma_{u}} s_{i j} \\
\sigma_{u}=\frac{1}{\sqrt{2}} \sqrt{\left(\sigma_{r r}-\sigma_{\theta \theta}\right)^{2}+\left(\sigma_{\theta \theta}-\sigma_{z z}\right)^{2}+\left(\sigma_{z z}-\sigma_{r r}\right)^{2}+6\left(\sigma_{z \theta}^{2}+\sigma_{\theta z}^{2}+\sigma_{z r}^{2}\right)} .
\end{gathered}
$$

В рассматриваемом плоском деформированном состоянии скорость осевой деформации ползучести $\dot{p}_{z z}$ принимается равной нулю:

$$
\dot{p}_{z z}=0 \text {. }
$$

Далее всюду через $H_{i}$ будем обозначать толщину мембраны на $i$-той стадии, $i=1,2,3,4$.

Примем, как обычно, для тонкостенных цилиндрических оболочек равенство

$$
\sigma_{r r}=0 \text {. }
$$

В этом случае из гипотезы пропорциональности девиаторов напряжений и скоростей деформаций ползучести (10) при учете (11) следует

$$
\sigma_{z z}=0.5 \sigma_{\theta \theta}, \quad \sigma_{u}=\frac{\sqrt{3}}{2} \sigma_{\theta \theta}=\frac{\sqrt{3}}{2} \frac{q \rho}{H_{0} H_{2}} .
$$


Рассматривая два близких деформированных состояния мембраны и учитывая, что деформированное состояние однородное, определим приращение окружной деформации ползучести:

$$
d p_{\theta \theta}=\frac{(\rho+d \rho)(\alpha+d \alpha)-\rho \alpha}{\rho \alpha}=\frac{d \rho}{\rho}+\frac{d \alpha}{\alpha} .
$$

Следовательно, скорость окружной деформации ползучести выражается формулой

$$
\dot{p}_{\theta \theta}=\frac{\dot{\rho}}{\rho}+\frac{\dot{\alpha}}{\alpha}
$$

Поскольку

$$
\rho \sin \alpha=1
$$

имеем

$$
\dot{\rho} \sin \alpha+\rho \dot{\alpha} \cos \alpha=0 .
$$

Поэтому выражение (12) преобразуется к виду

$$
\dot{p}_{\theta \theta}=\left(\frac{1}{\alpha}-\operatorname{ctg} \alpha\right) \dot{\alpha} .
$$

Из условия несжимаемости в случае плоского деформированного состояния получаем:

$$
\begin{gathered}
\dot{p}_{r r}+\dot{p}_{\theta \theta}+\dot{p}_{z z}=0, \quad \dot{p}_{z z}=0, \quad \dot{p}_{r r}=-\dot{p}_{\theta \theta} \\
\dot{p}_{u}=\frac{\sqrt{2}}{3} \sqrt{\left(\dot{p}_{r r}-\dot{p}_{\theta \theta}\right)^{2}+\left(\dot{p}_{\theta \theta}-\dot{p}_{z z}\right)^{2}+\left(\dot{p}_{z z}-\dot{p}_{r r}\right)^{2}+\dot{p}_{r \theta}^{2}+\dot{p}_{\theta z}^{2}+\dot{p}_{z r}^{2}} \\
\dot{p}_{u}=\frac{2}{\sqrt{3}} \dot{p}_{\theta \theta} .
\end{gathered}
$$

Так как скорость радиальной деформации ползучести выражается формулой

$$
\dot{p}_{r r}=-\frac{\dot{H}_{2}}{H_{2}}
$$

согласно равенству (14) получаем

$$
-\frac{\dot{H}_{2}}{H_{2}}=\left(\frac{1}{\alpha}-\operatorname{ctg} \alpha\right) \dot{\alpha} .
$$

Проинтегрируем уравнение (16) при начальных условиях $t=0, \alpha=\alpha_{1}$, $H_{2}=H_{1}$ :

$$
H_{2}=\frac{\sin \alpha}{\alpha} F, \quad F=\frac{\alpha_{1}}{\sin \alpha_{1}} .
$$

Полученные выражения (2), (13) и (17) позволяют представить окружное напряжение $\sigma_{\theta \theta}$ и интенсивность напряжений $\sigma_{u}$ в зависимости от угла раствора $\alpha$ :

$$
\sigma_{\theta \theta}=\frac{q \rho}{H_{2} H_{0}}=\frac{q \alpha}{F H_{0} \sin ^{2} \alpha}, \quad \sigma_{u}=\frac{\sqrt{3}}{2}\left|\sigma_{\theta \theta}\right|=\frac{\sqrt{3}}{2} \frac{q \alpha}{F H_{0} \sin ^{2} \alpha} .
$$


Из (15) при учете (1), (14) и (18) получаем зависимость угла раствора $\alpha$ от времени $t$ :

$$
t(\alpha)=\frac{2}{\sqrt{3}} \int_{\alpha_{1}}^{\alpha}\left(\frac{1}{\alpha}-\operatorname{ctg} \alpha\right)\left(\frac{2 F H_{0} \sin ^{2} \alpha}{\sqrt{3} q \alpha}\right)^{n} d \alpha, \quad t_{2}=t\left(\alpha_{2}\right) .
$$

При исследовании второй стадии деформирования мембраны угол $\alpha$ изменяется в диапазоне от $\alpha=\alpha_{1}$ до $\alpha_{2}=0.5 \pi$. Так как угол $\alpha_{1}$ удовлетворяет условию $\alpha_{1} \ll 0.5 \pi$ (т.е. постоянная $F$ незначительно отличается от единицы), упростим соотношение (17) и введем дополнительно зависимость $H_{2}(\alpha)$ во время второй стадии деформирования мембраны в виде

$$
H_{2}=\frac{\sin \alpha}{\alpha} .
$$

В конце второй стадии $\left(t=t_{2}\right)$ в случае прямоугольной матрицы раствор мембраны $2 \alpha\left(t_{2}\right)=2 \alpha_{2}$ удовлетворяет равенству $2 \alpha_{2}=\pi$. Так как значения $H_{2}$ при $F$ из (17) и $F=1$ практически совпадают (и последующие вычисления подтвердят это), далее под значением $t_{2}(\alpha)$ будем понимать соответствующее значение при $F=1$. В связи с этим момент времени $t_{2}$, при котором происходит окончание второй стадии, и толщина мембраны $H_{2}^{0}=H\left(t_{2}\right)$, вычисляемая согласно зависимости (19), определяются из

$$
t_{2}=t\left(\alpha_{2}\right), \quad H_{2}^{0}=H_{2}\left(t_{2}\right)=\frac{\sin \alpha_{2}}{\alpha_{2}}=\frac{2}{\pi} .
$$

Далее рассматривается ползучесть мембраны внутри жесткой матрицы при различных контактных условиях.

\section{3. Идеальное скольжение мембраны вдоль сторон матрицы}

3.1. Третья стадия. Рассмотрим ползучесть мембраны внутри длинной жесткой матрицы, сечение которой имеет форму прямоугольника шириной $2 a$ и высотой $b$. Введем дополнительные безразмерные параметры:

$$
\bar{b}=\frac{b}{a}, \quad \bar{x}_{0}=\frac{x_{0}}{a}, \quad \bar{y}_{0}=\frac{y_{0}}{a} .
$$

Далее черточки над этими безразмерными переменными также будем опускать.

Решение задачи имеет различный характер для относительно высокой матрицы $(b \geqslant 1)$ и относительно низкой матрицы $(b<1)$. Для определенности здесь будет рассмотрена ползучесть мембраны внутри относительно высокой матрицы.

В связи с осевой симметрией мембраны и матрицы далее рассматривается ползучесть правой половины мембраны в координатах $0 \leqslant x \leqslant 1,0 \leqslant y \leqslant b$ (рис. 2).

Свободное деформирование мембраны было рассмотрено в предыдущих пп. 1 и 2. В некоторый момент времени $\left(t=t_{2}\right)$ мембрана соприкасается с боковой стенкой матрицы. На этом свободное деформирование заканчивается, 

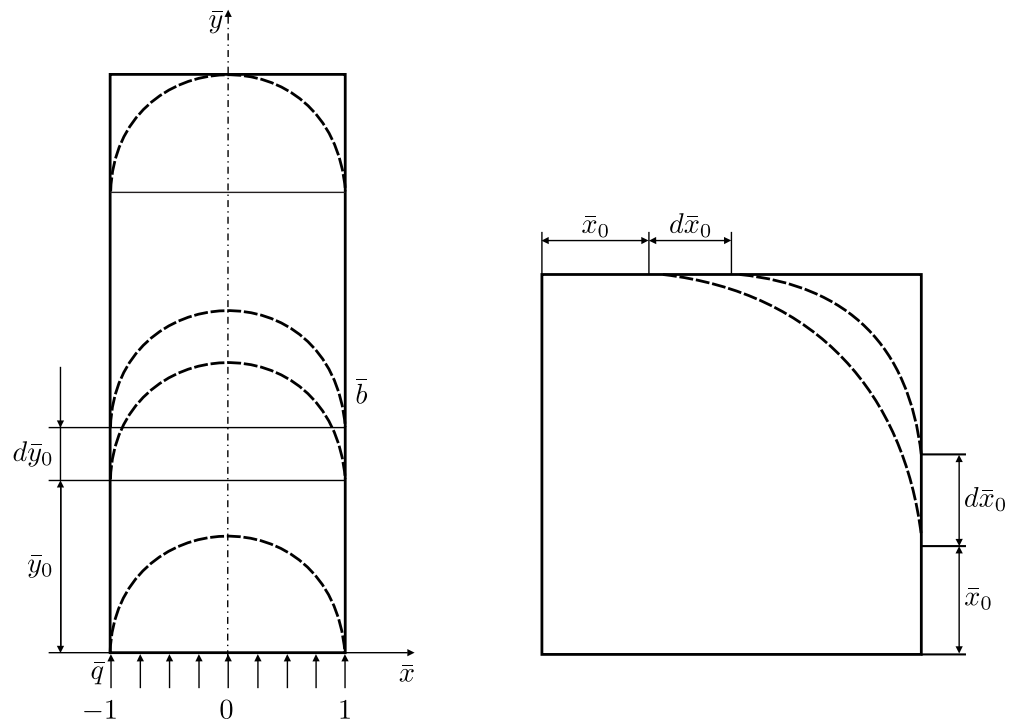

Рис. 2. Третья и четвертая стадии (идеальное скольжение и прилипание)

[Figure 2. The scheme for calculating the third and fourth stages of membrane deformation (ideal sliding and adhesion)]

и в дальнейшем при $t>t_{2}$ часть поверхности мембраны прилегает к внутренней поверхности матрицы.

При исследовании третьей стадии ползучести мембраны выделим два близких деформированных состояния с радиусом свободной дуги мембраны $\rho=1$ : одно характеризуется длиной участка контакта $y_{0}$, а другое - длиной участка контакта $\left(y_{0}+d y_{0}\right)$. Согласно определению $\dot{p}_{\theta \theta}$ имеем следующее:

$$
\begin{gathered}
d p_{\theta \theta}=\frac{\left(y_{0}+d y_{0}+0.5 \pi\right)-\left(y_{0}+0.5 \pi\right)}{y_{0}+0.5 \pi}=\frac{d y_{0}}{y_{0}+0.5 \pi}, \\
\dot{p}_{\theta \theta}=-\frac{1}{H_{3}} \frac{d H_{3}}{d t}, \quad d p_{\theta \theta}=-\frac{d H_{3}}{H_{3}} \\
p_{\theta \theta}=-\int_{H_{2}^{0}}^{H_{3}\left(y_{0}\right)} \frac{d H_{3}}{H_{3}}=\int_{0}^{y_{0}} \frac{d y_{0}}{y_{0}+0.5 \pi}=\ln \frac{H_{2}^{0}}{H_{3}\left(y_{0}\right)}=\ln \frac{y_{0}+0.5 \pi}{0.5 \pi} \\
H_{3}\left(y_{0}\right)=0.5 \pi \frac{H_{2}^{0}}{y_{0}+0.5 \pi}=\frac{1}{y_{0}+0.5 \pi} .
\end{gathered}
$$

Окончание третьей стадии происходит при касании мембраной верхней стенки матрицы, т.е. при $y_{0}=b-1$.

Толщина мембраны в конце третьей стадии определяется выражением

$$
H_{3}^{0}=H_{3}\left(t_{3}\right)=\frac{1}{b-1+0.5 \pi} .
$$

Таким образом,

$$
p_{\theta \theta}=\ln \frac{y_{0}+0.5 \pi}{0.5 \pi}, \quad \dot{p}_{\theta \theta}=\frac{1}{y_{0}+0.5 \pi} \frac{d y_{0}}{d t}, \quad \dot{p}_{u}=\frac{2}{\sqrt{3}} \frac{1}{y_{0}+0.5 \pi} \frac{d y_{0}}{d t} .
$$


Интенсивность напряжений определяется соотношением (2):

$$
\sigma_{u}\left(y_{0}\right)=\frac{\sqrt{3}}{2} \frac{q \rho}{H_{0} H_{3}\left(y_{0}\right)}, \quad \rho=1
$$

Подставляя выражения для $\dot{p}_{u}$ и $\sigma_{u}$ в $(1)$, получаем время окончания третьей стадии (при $\left.y_{0}=b-1\right)$ :

$$
\begin{gathered}
t_{3}\left(y_{0}\right)=t_{2}+\frac{2}{\sqrt{3}} \int_{0}^{y_{0}}\left[\frac{2 H_{0} H_{3}\left(y_{0}\right)}{\sqrt{3} q}\right]^{n} \frac{d y_{0}}{y_{0}+0.5 \pi} \\
t_{3}=t_{2}+\left(\frac{2}{\sqrt{3}}\right)^{(n+1)}\left(\frac{H_{0}}{q}\right)^{n} \frac{1}{n}\left[\frac{1}{(0.5 \pi)^{n}}-\frac{1}{(b-1+0.5 \pi)^{n}}\right] .
\end{gathered}
$$

3.2. Четвертая стадия. На четвертой стадии ползучесть мембраны при касании ею обеих сторон матрицы описывается аналогично (рис. 2):

$$
\begin{gathered}
d p_{\theta \theta}=F\left(x_{0}\right) d x_{0}, \quad F\left(x_{0}\right)=\frac{2-0.5 \pi}{b-1+0.5 \pi+(2-0.5 \pi) x_{0}}, \\
p_{\theta \theta}=-\int_{H_{3}^{0}}^{H_{4} x_{0}} \frac{d H_{4}}{H_{4}}=\int_{0}^{x_{0}} F\left(x_{0}\right) d x_{0}=\ln \frac{H_{3}^{0}}{H_{4}\left(x_{0}\right)}= \\
=\ln \frac{b-1+0.5 \pi+x_{0}(2-0.5 \pi)}{b-1+0.5 \pi}, \\
H_{4}\left(x_{0}\right)=H_{3}^{0} \frac{b-1+0.5 \pi}{b-1+0.5 \pi+(2-0.5 \pi) x_{0}}=\frac{1}{b-1+0.5 \pi+(2-0.5 \pi) x_{0}} .
\end{gathered}
$$

Конец четвертой стадии происходит при значении $x_{0}=x_{0}^{0}$, удовлетворяющем неравенству $1-x_{0}^{0} \ll 1$.

Толщина мембраны в конце четвертой стадии определяется выражением

$$
H_{4}^{0}\left(x_{0}^{0}\right)=\frac{1}{b-1+0.5 \pi+(2-0.5 \pi) x_{0}^{0}} .
$$

Интенсивность напряжений определяется следующим соотношением:

$$
\begin{aligned}
\sigma_{u}\left(x_{0}\right) & =\frac{\sqrt{3}}{2} \frac{q \rho}{H_{0} H\left(x_{0}\right)}, \quad \rho=1-x_{0} \\
\sigma_{u}\left(x_{0}\right) & =\frac{\sqrt{3}}{2} \frac{q}{H_{0}}\left[b-1+0.5 \pi+(2-0.5 \pi) x_{0}\right]\left(1-x_{0}\right) .
\end{aligned}
$$

Интенсивность скоростей деформаций ползучести согласно (20) определяется формулой

$$
\dot{p}_{u}=\frac{2}{\sqrt{3}} F\left(x_{0}\right) \frac{d x_{0}}{d t}=\frac{2}{\sqrt{3}} \frac{2-0.5 \pi}{b-1+0.5 \pi+(2-0.5 \pi) x_{0}} \frac{d x_{0}}{d t} .
$$


Подставляя (20) и (21) в (1), получаем

$$
\begin{aligned}
\frac{\sqrt{3}}{2} \frac{2-0.5 \pi}{b-1+0.5 \pi+(2-0.5 \pi) x_{0}} & \frac{d x_{0}}{d t}= \\
= & \frac{\sqrt{3}}{2} \frac{q}{H_{0}}\left[b-1+0.5 \pi+(2-0.5 \pi) x_{0}\right]\left(1-x_{0}\right)^{n} .
\end{aligned}
$$

Отсюда

$$
\begin{aligned}
& t_{4}\left(x_{0}\right)=t_{3}+\frac{2}{\sqrt{3}} \int_{0}^{x_{0}}\left[\frac{2 H_{0} H_{4}\left(x_{0}\right)}{\sqrt{3} q\left(1-x_{0}\right)}\right]^{n} F\left(x_{0}\right) d x_{0}= \\
& =t_{3}+\left(\frac{2}{\sqrt{3}}\right)^{(n+1)}\left(\frac{H_{0}}{q}\right)^{n} \int_{0}^{x_{0}} \frac{(2-0.5 \pi) d x_{0}}{\left[b-1+0.5 \pi+(2-0.5 \pi) x_{0}\right]^{(n+1)}\left(1-x_{0}\right)^{n}} .
\end{aligned}
$$

Время практически полного заполнения матрицы мембраной определяется выражением (22) при замене $x_{0}$ на $x_{0}^{0}$ :

$$
t_{4}^{0}=t_{4}\left(x_{0}^{0}\right)
$$

\section{4. Прилипание мембраны вдоль сторон матрицы}

4.1. Третья стадия. При исследовании третьей стадии ползучести мембраны выделим два близких состояния с радиусом свободной дуги мембраны $\rho=1$. Согласно определению $\dot{p}_{\theta \theta}$ имеем:

$$
\begin{gathered}
p_{\theta \theta}=-\int_{H_{2}^{0}}^{H_{3}\left(y_{0}\right)} \frac{d H_{3}}{H_{3}}=\int_{0}^{y_{0}} \frac{2 d y_{0}}{\pi}=\frac{2}{\pi} y_{0}=\ln \frac{H_{2}^{0}}{H_{3}}, \quad H_{3}=H_{2}^{0} \exp \left(-\frac{2}{\pi} y_{0}\right), \\
\dot{p}_{u}=\frac{2}{\sqrt{3}} \dot{p}_{\theta \theta}=-\frac{\dot{H}_{3}}{H_{3}}=\frac{2}{\sqrt{3}} \frac{2}{\pi} \frac{d y_{0}}{d t} .
\end{gathered}
$$

Окончание третьей стадии происходит при касании верхней стенки матрицы, т.е. при $y_{0}=b-1$.

Толщина мембраны в конце третьей стадии определяется выражением

$$
H_{3}^{0}=H_{3}\left(t_{3}\right)=H_{2}^{0} \exp \left(-\frac{2}{\pi}(b-1)\right)=\frac{2}{\pi} \exp \left(-\frac{2}{\pi}(b-1)\right) .
$$

Интенсивность напряжений определяется следующим соотношением:

$$
\sigma_{u}=\frac{\sqrt{3}}{2} \frac{q \rho}{H_{3} H_{0}}, \quad \rho=1, \quad \sigma_{u}\left(y_{0}\right)=\frac{\sqrt{3}}{2} \frac{q}{H_{0}} \frac{\pi}{2} \exp \left(\frac{2}{\pi} y_{0}\right) .
$$

Подставляя (23) и (24) в (1), получаем

$$
t_{3}\left(y_{0}\right)=t_{2}+\left(\frac{4}{\sqrt{3} \pi}\right)^{(n+1)}\left(\frac{H_{0}}{q}\right)^{n} \int_{0}^{y_{0}} \exp ^{n}\left(-\frac{2}{\pi} y_{0}\right) d y_{0} .
$$


Время, при котором мембрана начинает касаться поперечной стороны матрицы, определяется с помощью (25) при $y_{0}=b-1$ :

$$
t_{3}^{0}=t_{3}(b-1)
$$

4.2. Четвертая стадия. На четвертой стадии ползучесть мембраны при касании ею продольной и поперечной сторон матрицы описывается аналогично:

$$
\begin{gathered}
d p_{\theta \theta}=\frac{2 d x_{0}+0.5 \pi\left(1-x_{0}-d x_{0}\right)-0.5 \pi\left(1-x_{0}\right)}{0.5 \pi\left(1-x_{0}\right)}=\frac{(2-0.5 \pi) d x_{0}}{0.5 \pi\left(1-x_{0}\right)} \\
p_{\theta \theta}=-\int_{H_{3}^{0}}^{H_{4}\left(x_{0}\right)} \frac{d H_{4}}{H_{4}}=\int_{0}^{x_{0}} \frac{(2-0.5 \pi) d x_{0}}{0.5 \pi\left(1-x_{0}\right)}=\ln \frac{H_{3}^{0}}{H_{4}\left(x_{0}\right)}=\frac{2-0.5 \pi}{0.5 \pi} \ln \frac{1}{1-x_{0}} \\
\frac{H_{3}^{0}}{H_{4}\left(x_{0}\right)}=\left(1-x_{0}\right)^{-\frac{2-0.5 \pi}{0.5 \pi}}, \quad H_{4}\left(x_{0}\right)=H_{3}^{0}\left(1-x_{0}\right)^{\frac{2-0.5 \pi}{0.5 \pi}}
\end{gathered}
$$

Окончание четвертой стадии происходит при $x=x_{0}^{0}$, удовлетворяющем неравенству $1-x_{0}^{0} \ll 1$.

Толщина мембраны в конце четвертой стадии определяется выражением

$$
H_{4}^{0}=H_{4}\left(x_{0}^{0}\right)
$$

Интенсивность напряжений определяется следующим соотношением:

$$
\sigma_{u}\left(x_{0}\right)=\frac{\sqrt{3}}{2} \frac{q \rho}{H_{4}\left(x_{0}\right) H_{0}}, \quad \rho=1-x_{0}, \quad \sigma_{u}\left(x_{0}\right)=\frac{\sqrt{3}}{2} \frac{q}{H_{0}} \frac{1-x_{0}}{H_{4}\left(x_{0}\right)} .
$$

Интенсивность скоростей деформаций ползучести определяется формулой

$$
\dot{p}_{u}=\frac{2}{\sqrt{3}} \frac{2-0.5 \pi}{0.5 \pi} \frac{1}{1-x_{0}} \frac{d x_{0}}{d t} .
$$

Подставляя (26) и (27) в (1), получаем

$$
\begin{gathered}
t_{4}\left(x_{0}\right)=t_{3}+\frac{2}{\sqrt{3}} \int_{0}^{x_{0}}\left[\frac{2 H_{4} H_{0}}{\sqrt{3} q\left(1-x_{0}\right)}\right]^{n} \frac{(2-0.5 \pi) d x_{0}}{0.5 \pi\left(1-x_{0}\right)}= \\
\quad=t_{3}+\left(\frac{2}{\sqrt{3}}\right)^{(n+1)}\left(\frac{H_{0}}{q}\right)^{n} \frac{2-0.5 \pi}{0.5 \pi} \frac{H_{4}^{n}\left(x_{0}\right)}{\left(1-x_{0}\right)^{(n+1)}} .
\end{gathered}
$$

Время практически полного заполнения матрицы в этом случае определяется выражением

$$
t_{4}^{0}=t_{4}\left(x_{0}^{0}\right)
$$




\section{5. Скольжение мембраны вдоль сторон матрицы с учетом трения}

В отличие от пп. 3 и 4, где были рассмотрены два крайних граничных условия - полное идеальное скольжение и прилипание мембраны вдоль стенок матрицы, здесь рассматривается решение, когда на границе контакта трение удовлетворяет закону Кулона.

Задача решается итерационным методом. Применяется аппроксимация производных по первому порядку точности. Произвольная $(k+1)$-я итерация (шаг) характеризуется приращением времени $d t^{k+1}$ и соответствующим этому приращению дополнительным участком свободной части мембраны $d\left(s_{k+1}^{k+1}\right)$, который начинает контактировать со стенками матрицы (рис. 3). Нижние цифровые индексы при параметрах, характеризующих отдельный участок, обозначают шаг, на котором произошло прилегание этого участка к матрице, а верхние индексы - шаг, на котором берутся значения этих параметров во времени. На границе мембраны и матрицы принимается закон трения Кулона с коэффициентом трения $\mu$. При этом известны следующие значения параметров, полученные на предыдущих шагах: $q^{1}, \ldots, q^{k} ; d t^{1}, \ldots, d t^{k} ; \rho_{0}^{k}, \ldots, \rho_{k}^{k}$; $d s_{1}^{k}, \ldots, d s_{k}^{k} ; H_{0}^{k}, \ldots, H_{k}^{k} ;\left(\sigma_{\theta \theta}\right)_{0}^{k}, \ldots,\left(\sigma_{\theta \theta}\right)_{k}^{k} ;\left(\dot{p}_{\theta \theta}\right)_{0}^{k}, \ldots,\left(\dot{p}_{\theta \theta}\right)_{k}^{k}$.

Здесь и в дальнейшем величины $\sigma_{\theta}, \dot{p}_{\theta}$ с соответствующими верхними и нижними индексами означают растягивающие напряжения на границе мембраны и матрицы на соответствующем шаге итерации.

Зададим $d t^{k+1}$ и $q^{k+1}$. На $(k+1)$-м шаге необходимо вычислить $d s_{k+1}^{k+1}$, $\left(\sigma_{\theta \theta}\right)_{k+1}^{k+1}, H_{k+1}^{k+1}$ и найти новые значения системы рассматриваемых величин.
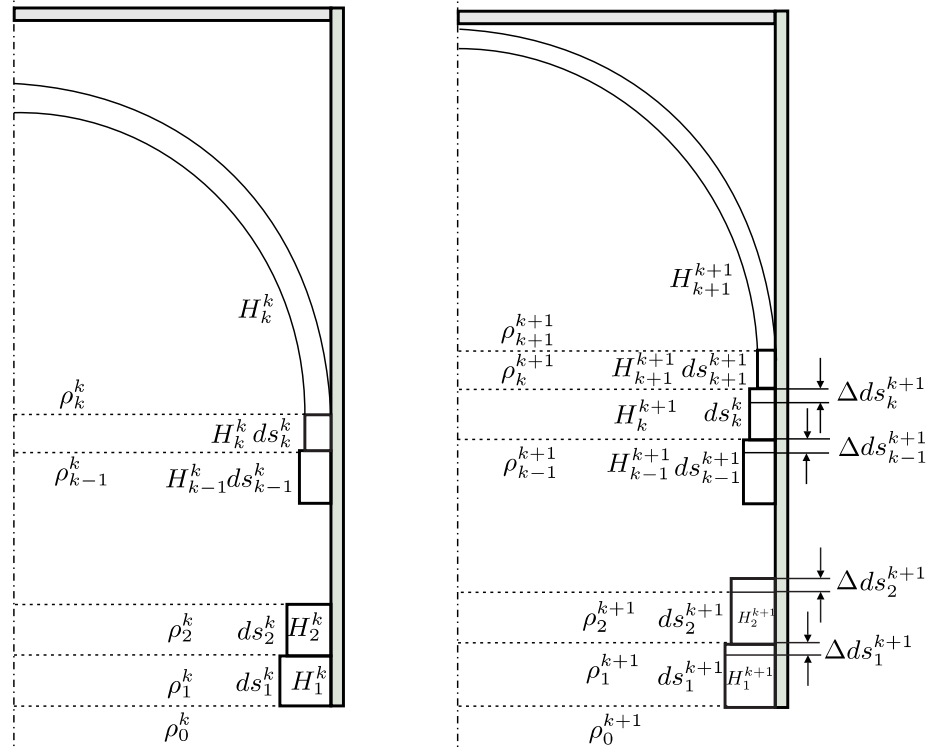

Рис. 3. Третья и четвертая стадии при учете трения

[Figure 3. The scheme for calculating the third and fourth stages of membrane deformation with considering friction] 
5.1. Третья стадия. Свободная часть дуги $\frac{\pi}{2} \rho=\frac{\pi}{2}$ за время $d t^{k+1}$ удлиняется под действием давления $q^{k+1}$ за счет ползучести:

$$
d s_{k+1}^{k+1}=\frac{\pi}{2} d t^{k+1} \dot{p}_{\theta \theta}=\frac{\pi}{2} d t^{k+1}\left(\frac{\sqrt{3} q^{k+1}}{2 H_{0} H_{k}^{k}}\right)^{n} .
$$

Соответственно, уменьшается толщина свободной части мембраны. Используя $\dot{p}_{\theta \theta}=-\dot{H} / H$, уравнение равновесия и выражение $(1)$, получаем

$$
H_{k+1}^{k+1}=H_{k}^{k}\left(1-\left(\frac{\sqrt{3}}{2}\left(\sigma_{\theta \theta}\right)_{k}^{k}\right)^{n} d t^{k+1}\right) .
$$

Новое значение действующего поперечного напряжения найдем из уравнения равновесия:

$$
\left(\sigma_{\theta \theta}\right)_{k+1}^{k+1}=\frac{q^{k+1}}{H_{0} H_{k+1}^{k+1}} .
$$

Будем считать, что напряжения на вновь прилипшем отрезке мембраны равны на верхней и нижней части:

$$
\left(\sigma_{\theta \theta}\right)_{k}^{k+1}=\left(\sigma_{\theta \theta}\right)_{k+1}^{k+1} .
$$

Далее производится вычисление новых значений $\left(\sigma_{\theta \theta}\right)_{i}^{k+1}, H_{i}^{k+1}$ через перерасчет напряжений на уже прилипших отрезках. При этом будем учитывать, что если сумма силы трения и силы, действующей на нижней части этого элемента мембраны, превышает силу, действующую на верхней части элемента, то данный элемент мембраны считается прилипшим и поэтому он не деформируется на стадии $(k+1)$, иначе он деформируется с учетом трения о стенки матрицы.

Сила трения на $i$-том кусочке определяется выражением

$$
\left(F_{\mathrm{fr}}\right)_{i}=\mu q^{k+1} d s_{i}^{k}
$$

Если неравенство

$$
\left(F_{\mathrm{fr}}\right)_{i}+\left(\sigma_{\theta \theta}\right)_{i-1}^{k} H_{0} H_{i}^{k} \geqslant\left(\sigma_{\theta \theta}\right)_{i}^{k+1} H_{0} H_{i}^{k}
$$

выполнено, то размеры этого элемента мембраны и значения соответствующих напряжений сохраняются:

$$
d s_{i}^{k+1}=d s_{i}^{k}, \quad H_{i}^{k+1}=H_{i}^{k}, \quad\left(\sigma_{\theta \theta}\right)_{i-1}^{k+1}=\left(\sigma_{\theta \theta}\right)_{i-1}^{k}, \quad \Delta d s_{i}^{k+1}=0,
$$

в противном случае отрезок растягивается, и его новые значения вычисляются по соотношениям

$$
\begin{gathered}
d s_{i}^{k+1}=\Delta d s_{i}^{k+1}+d s_{i}^{k}, \quad \Delta d s_{i}^{k+1}=d s_{i}^{k}\left(\frac{\sqrt{3}}{4}\left(\left(\sigma_{\theta \theta}\right)_{i-1}^{k}+\left(\sigma_{\theta \theta}\right)_{i}^{k}\right)\right)^{n} d t^{k+1}, \\
\left(\sigma_{\theta \theta}\right)_{i-1}^{k+1}=\left(\sigma_{\theta \theta}\right)_{i}^{k+1}-\frac{\left(F_{\mathrm{fr}}\right)_{i}}{H_{0} H_{i}^{k}} \\
H_{i}^{k+1}=H_{i}^{k}\left(1-\left(\frac{\sqrt{3}}{2}\left(\sigma_{\theta \theta}\right)_{i}^{k}\right)^{n}\right) d t^{k+1} .
\end{gathered}
$$

Окончание расчета в рамках третьей стадии наступает, если выполнено одно из двух условий: 
- условие касания верхней части матрицы $\sum_{i} d s_{i}^{k+1} \geqslant b-1$;

- условие разрушения мембраны $H_{k+1}^{k+1} \leqslant 0$.

Значения $t_{3}, H_{3}, \sigma_{3}$ будут характеризовать значения времени, толщины и напряжения в конце третьей стадии деформирования мембраны.

5.2. Четвертая стадия. Расчет для четвертой стадии деформирования мембраны проводится аналогично расчету третьей стадии по шагам. Из-за высокой нелинейности задачи в начальный момент времени на четвертой стадии и практически неизменно в последующем, для получения хорошего результата расчета используется неравномерный шаг по времени. В начальный момент времени шаг очень мал, далее расчет проводится с повышением значения шага до больших значений на финальном этапе деформирования. Для этого используется расчет шага по квадратичной функции: $d t \sim t^{2}$. Для того чтобы получить решение за $n$ шагов от времени $t_{3}$ до $t_{4}$, необходимо, чтобы было выполнено следующее условие:

$$
\sum_{i}^{n} d t^{(i)}=t_{4}-t_{3}
$$

Используя квадратичную зависимость шага по времени от времени, получаем

$$
d t^{(i)}=B\left((i+1)^{3}-i^{3}\right), \quad B=\frac{t_{4}-t_{3}}{n(n-1)^{2}} .
$$

На $k$-том шаге вычисляются значения параметров шага $(k+1)$. Из уравнения равновесия вычисляются новые значения продольных напряжений:

$$
\left(\sigma_{\theta \theta}\right)_{k+1}^{k+1}=\frac{\rho^{k} q^{k}}{H_{0} H_{k}^{k}}, \quad\left(\sigma_{\theta \theta}\right)_{k}^{k+1}=\left(\sigma_{\theta \theta}\right)_{k+1}^{k+1} .
$$

Толщина нового элемента на четвертой стадии вычисляется аналогично третьей стадии по соотношению

$$
H_{k+1}^{k+1}=H_{k}^{k}\left(1-\left(\frac{\sqrt{3}}{2}\left(\sigma_{\theta \theta}\right)_{k}^{k}\right)^{n} d t^{k+1}\right) .
$$

На четвертой стадии имеются два набора прилипших элементов: элементы, прилипшие на третьей стадии $\left(d \hat{s}_{i}^{k}, \hat{H}_{i}^{k},\left(\hat{\sigma}_{\theta \theta}\right)_{i}^{k}\right)$ и элементы, прилипающие на четвертой стадии $\left(d s_{i}^{k}, H_{i}^{k},\left(\sigma_{\theta \theta}\right)_{i}^{k}\right)$ (без дополнительных обозначений). Они рассматриваются отдельно в соответствии с выражениями (28)-(30) с помощью предварительных вычислений $d \hat{s}_{i}^{k+1}, \hat{H}_{i}^{k+1},\left(\hat{\sigma}_{\theta \theta}\right)_{i}^{k+1}, \Delta d \hat{s}_{i}^{k+1}$ и последующих вычислений $d s_{i}^{k+1}, H_{i}^{k+1},\left(\sigma_{\theta \theta}\right)_{i}^{k+1}, \Delta d s_{i}^{k+1}$.

Для вычисления удлинения свободной части мембраны рассмотрим два близких состояния и согласно определению $\dot{p}_{\theta \theta}$ учтем, что прилипание мембраны к стенкам матрицы происходит симметрично сбоку и сверху матрицы, поэтому будем рассматривать только четверть мембраны с углом раствора $\pi / 4$, в результате имеем

$$
d \dot{p}_{\theta \theta}=\frac{\alpha d \rho^{k+1}+d s_{k+1}^{k+1}}{\alpha \rho^{k}}
$$


Согласно геометрическим условиям, изменение радиуса составляет

$$
d \rho^{k+1}=-\left(d s_{k+1}^{k+1}+D s^{k+1}+0.5 D \hat{s}^{k+1}\right)
$$

где

$$
D s^{k+1}=\sum_{i} d s_{i}^{k+1}, \quad D \hat{s}^{k+1}=\sum_{i} d \hat{s}_{i}^{k+1} .
$$

Тогда

$$
\begin{aligned}
d \dot{p}_{\theta \theta}=\frac{-\alpha\left(d s_{k+1}^{k+1}+D s^{k+1}+0.5 D \hat{s}^{k+1}\right)}{\alpha \rho^{k}}+d s_{k+1}^{k+1} & = \\
& =d s_{k+1}^{k+1} \frac{1-\alpha}{\alpha \rho^{k}}-\frac{D s^{k+1}+0.5 D \hat{s}^{k+1}}{\rho^{k}} .
\end{aligned}
$$

Отсюда, используя выражение (1), получаем значение вновь прилипшего отрезка мембраны при подстановке $\alpha=\pi / 4$ :

$$
d s_{k+1}^{k+1}=\frac{\pi \rho_{k}^{k}}{4-\pi}\left[\left(\frac{\sqrt{3} q^{k+1} \rho^{k}}{2 H_{0} H_{k+1}^{k+1}}\right)^{n} d t^{k+1}+\frac{D s^{k+1}+0.5 D \hat{s}^{k+1}}{\rho^{k}}\right] .
$$

Новое значение радиуса мембраны

$$
\rho^{k+1}=\rho^{k}-d s_{k+1}^{k+1}-D s^{k+1}-D \hat{s}^{k+1} .
$$

Длина контактирующей части мембраны вычисляется из выражения

$$
s^{k+1}=D s^{k+1}+D \hat{s}^{k+1}=\sum_{i} d s_{i}^{k+1}+\sum_{i} d \hat{s}_{i}^{k+1} .
$$

Окончание расчета в рамках четвертой стадии наступает, если будет выполнено одно из двух условий:

- условие заполнение матрицы $\rho \leqslant x_{0}^{0}$,

- условие разрушения мембраны $H_{k+1}^{k+1} \leqslant 0$.

Полученные в этот момент времени значения $\sum_{i}^{n} d t^{i}, H_{k+1}^{k+1},\left(\sigma_{\theta \theta}\right)_{k+1}^{k+1}$ характеризуются значениями $t_{4}, H_{4}, \sigma_{4}$ в конце четвертой стадии.

\section{6. Ползучесть мембраны при постоянном давлении $q$}

В качестве примера рассмотрим деформирование мембраны высотой $b=2$ со следующими выбранными параметрами:

$$
H_{0}=0.01, \quad n=3, \quad x_{0}^{0}=0.99, \quad k=100 .
$$

В качестве величин поперечного давления примем два значения $q$, различающихся в 5 раз: $q=0.5 \cdot 10^{-4}$ и $q=2.5 \cdot 10^{-4}$, в этом случае значения угла $\alpha_{1}$, полученные при мгновенном приложении внешнего давления $q$, равны соответственно $\alpha_{1}=0.061$ и $\alpha_{1}=0.104$. Так как значения отношения $F$ в $(17)$ очень незначительно отличаются от 1 (соответственно $F=1.001$ и $F=1.002$ ), замена соотношения (17) на (19) является вполне обоснованной. 
На рис. 4 и 5 соответственно приведены зависимости толщины мембраны $H(t)$ и интенсивности напряжений $\sigma_{u}(t)$ на свободной части мембраны от времени $t$ для всех четырех стадий деформирования при постоянном давлении $q=0.5 \cdot 10^{-4}$. Для стадий стесненного деформирования (третья и четвертая стадии) зависимости $H(t)$ и $\sigma_{u}(t)$ приведены для идеального скольжения (сплошная линия), прилипания (штрих-пунктирная линия), расчет с учетом трения при коэффициенте трения $\mu=0.1$ (пунктирная линия) и коэффициенте трения $\mu=0.3$ (штриховая линия). На рис. 6 и 7 соответственно приведены аналогичные зависимости $H(t)$ и $\sigma_{u}(t)$ при постоянном давлении $q=2.5 \cdot 10^{-4}$. Значения основных параметров $t, H, \sigma_{u}$ в конце каждой стадии приведены в табл. 1 и 2 для идеального скольжения и прилипания и в табл. 3 и 4 для $\mu=0.1$ и $\mu=0.3$ (колонки $A$ при $q=0.5 \cdot 10^{-4}$ и $B$ при $q=2.5 \cdot 10^{-4}$ ).

Из табл. 1, 2 следует, что значения толщин свободной части мембраны в конце каждой стадии при $q=0.5 \cdot 10^{-4}$ и $q=2.5 \cdot 10^{-4}$ совпадают, а времена деформирования различаются в 125 раз. При этом при учете трения значения толщин свободной части мембраны незначительно отличаются, при этом для меньшего значения $q$ толщина в конце стадии меньше, что может объясняться тем, что мембрана при меньшем давлении больше прилипает к стенкам матрицы, соответственно, растяжение происходит за счет более интенсивного утонения свободной части.

Во всех рассмотренных случаях зависимости $H(t)$ являются монотонно убывающими функциями времени $t$. Расчеты показывают, что интенсивности напряжений $\sigma_{u}(t)$ на второй и третьей стадиях возрастают, а на четвертой стадии убывают. Толщины свободной части мембраны в конце деформирования при прилипании меньше, чем при идеальном скольжении, а интенсивности напряжений, наоборот, больше, а при учете трения данные значения лежат между этими величинами.

Вычисления показывают, что при заданных значениях используемых параметров основная длительность деформирования мембраны вплоть до практически полного заполнения соответствует четвертой стадии $\left(t_{4}^{0}-t_{3}^{0}\right)$, длительность третьей стадии минимальна: $\left(t_{3}^{0}-t_{2}^{0}\right)<\left(t_{4}^{0}-t_{3}^{0}\right)$.

\section{7. Ползучесть мембраны при кусочно-постоянном давлении $q$}

Рассмотрим две программы нагружения. В первой программе (см. колонки $C$ в табл. 1,2$)$ сначала деформирование происходит при $q^{(1)}=0.5 \cdot 10^{-4}$ в течение времени $\tau_{1}$, равного половине соответствующей длительности $\tau_{1}^{0}$ заполнения мембраны (т.е. $\tau_{1}=0.5 \tau_{1}^{0}$ ), затем величина $q$ скачкообразно изменяется и становится равной $q^{(2)}=2.5 \cdot 10^{-4}$, при $\tau>\tau_{1}$ рассматривается ползучесть вплоть до заполнения матрицы при $t=t^{* 1}$. Во второй программе нагружения (колонка $D$ в табл. 1, 2) сначала мембрана деформируется при $q^{(2)}=2.5 \cdot 10^{-4}$ в течение $0 \leqslant t \leqslant 0.5 \tau_{2}^{0}$ (где $\tau_{2}^{0}$ - длительность заполнения матрицы при $q^{(2)}=$ const), а затем мембрана деформируется при $q^{(1)}=0.5 \cdot 10^{-4}$ вплоть до заполнения матрицы при $t=t^{* 2}$.

Согласно табл. 1 и 2 , при данных значениях $q^{(1)}$ и $q^{(2)}$ скачкообразное изменение $q$ происходит в процессе 4-й стадии первоначального значения $q$.

Применим к этой задаче правило суммирования парциальных времен $S$, определяемое как сумма отношений длительностей ползучести при постоян- 


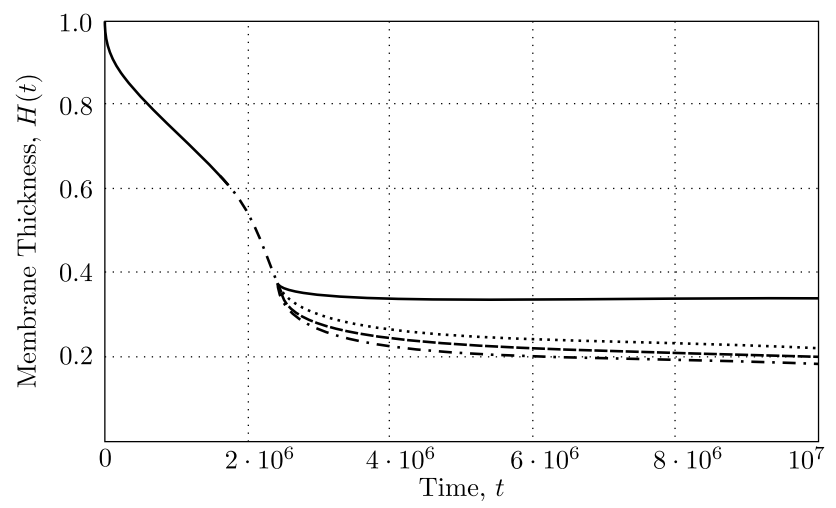

Рис. 4. Зависимости $H(t)$ при $q=0.5 \cdot 10^{-4}$

[Figure 4. The membrane thickness at $q=0.5 \cdot 10^{-4}$ : ideal sliding (solid line), adhesion (dash-dotted line), calculation taking into account friction with the friction coefficient $\mu=0.1$ (dotted line) and $\mu=0.3$ (dashed line)]

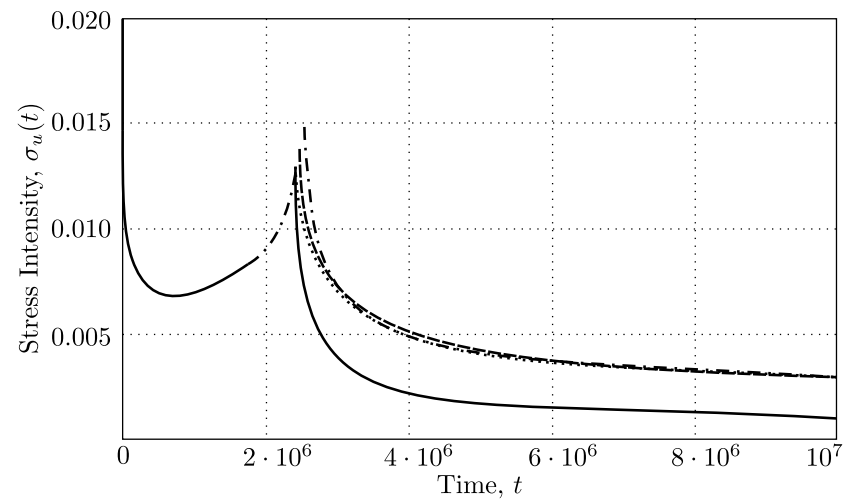

Рис. 5. Зависимости $\sigma_{u}(t)$ при $q=0.5 \cdot 10^{-4}$

[Figure 5. The stress intensity at $q=0.5 \cdot 10^{-4}$ : ideal sliding (solid line), adhesion (dash-dotted line), calculation taking into account friction with the friction coefficient $\mu=0.1$ (dotted line) and $\mu=0.3$ (dashed line)]

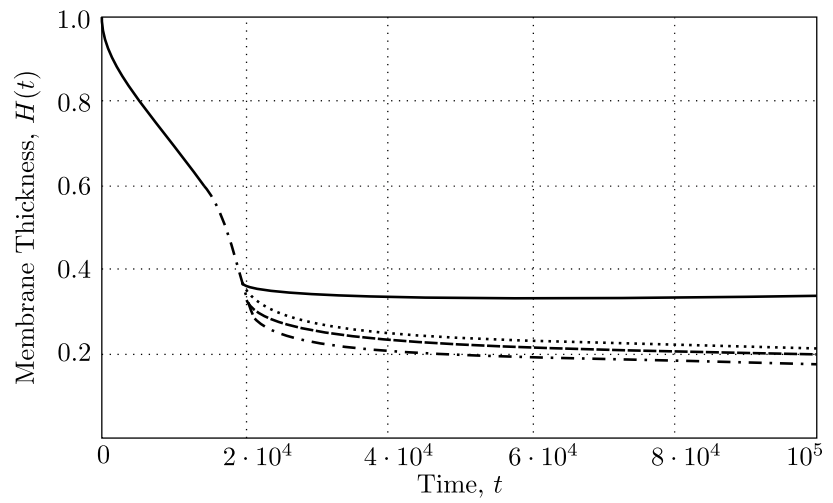

Рис. 6. Зависимости $H(t)$ при $q=2.5 \cdot 10^{-4}$

[Figure 6. The membrane thickness at $q=2.5 \cdot 10^{-4}$ : ideal sliding (solid line), adhesion (dash-dotted line), calculation taking into account friction with the friction coefficient $\mu=0.1$ (dotted line) and $\mu=0.3$ (dashed line)] 

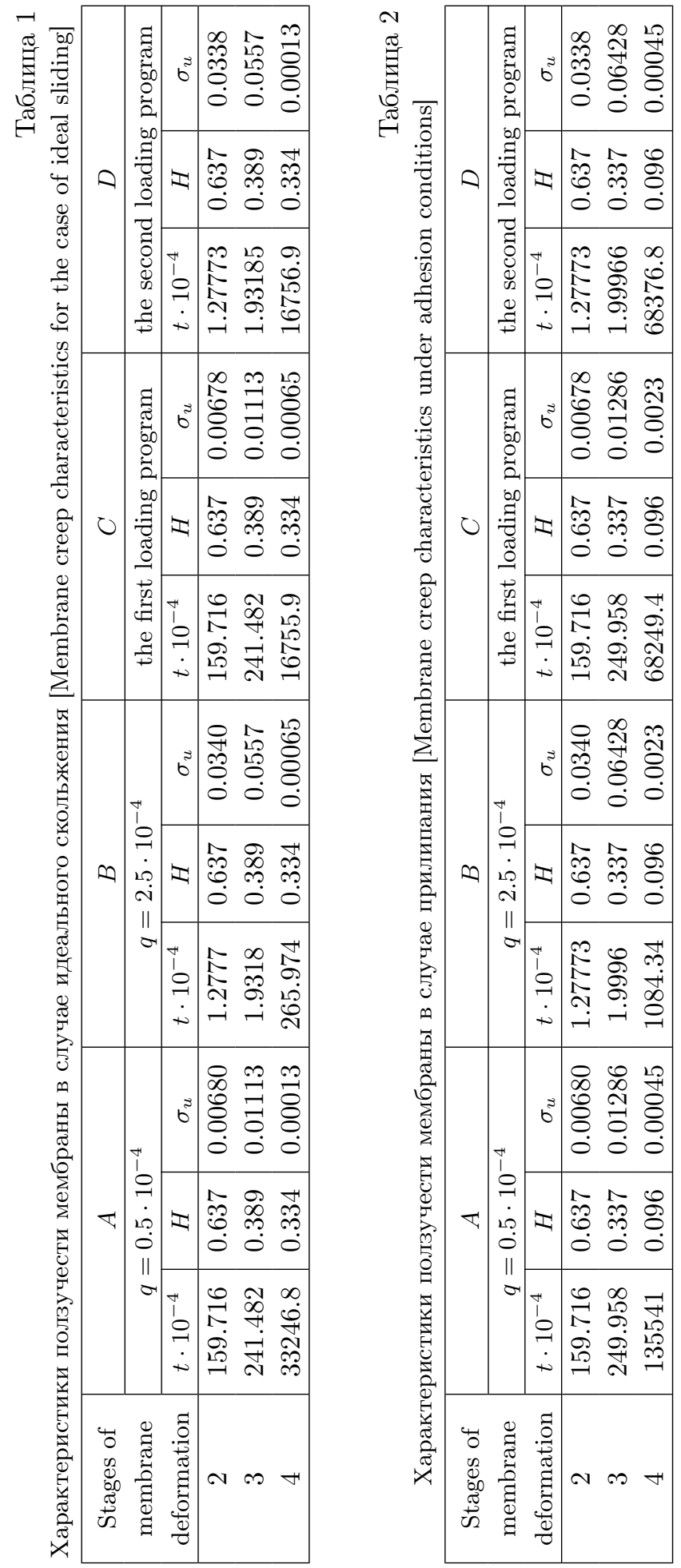


\section{Таблица 3}

Характеристики ползучести мембраны в случае учета трения о стенки матрицы, коэффициент трения $\mu=0.1$ [Creep characteristics of the membrane under the friction against the matrix walls; the coefficient of friction is $\mu=0.1$ ]

\begin{tabular}{|c|c|c|c|c|c|c|}
\hline \multirow{2}{*}{$\begin{array}{c}\text { Stages of } \\
\text { membrane }\end{array}$} & \multicolumn{3}{|c|}{$A$} & \multicolumn{3}{|c|}{$B$} \\
\cline { 2 - 7 } deformation & \multicolumn{2}{|c|}{$q=0.5 \cdot 10^{-4}$} & \multicolumn{3}{|c|}{$q=2.5 \cdot 10^{-4}$} \\
\cline { 2 - 7 } & $t \cdot 10^{-4}$ & $H$ & $\sigma_{u}$ & $t \cdot 10^{-4}$ & $H$ & $\sigma_{u}$ \\
\hline 2 & 159.716 & 0.637 & 0.00680 & 1.2777 & 0.637 & 0.0340 \\
3 & 242.171 & 0.386 & 0.01124 & 1.9391 & 0.385 & 0.0560 \\
4 & 102343 & 0.110 & 0.00039 & 834.47 & 0.110 & 0.0020 \\
\hline
\end{tabular}

Таблица 4

Характеристики ползучести мембраны в случае учета трения о стенки матрицы, коэффициент трения $\mu=0.3$ [Creep characteristics of the membrane under the friction against the matrix walls; the coefficient of friction is $\mu=0.3$ ]

\begin{tabular}{|c|c|c|c|c|c|c|}
\hline \multirow{2}{*}{$\begin{array}{c}\text { Stages of } \\
\text { membrane } \\
\text { deformation }\end{array}$} & \multicolumn{3}{|c|}{$A$} & \multicolumn{3}{|c|}{$B$} \\
\cline { 2 - 7 } & \multicolumn{2}{|c|}{$q=0.5 \cdot 10^{-4}$} & \multicolumn{3}{|c|}{$q=2.5 \cdot 10^{-4}$} \\
\cline { 2 - 7 } & $t \cdot 10^{-4}$ & $H$ & $\sigma_{u}$ & $t \cdot 10^{-4}$ & $H$ & $\sigma_{u}$ \\
\hline 2 & 159.716 & 0.637 & 0.00680 & 1.2777 & 0.637 & 0.0340 \\
3 & 243.373 & 0.378 & 0.01143 & 1.9524 & 0.377 & 0.0574 \\
4 & 114129 & 0.108 & 0.00040 & 957.31 & 0.107 & 0.0020 \\
\hline
\end{tabular}

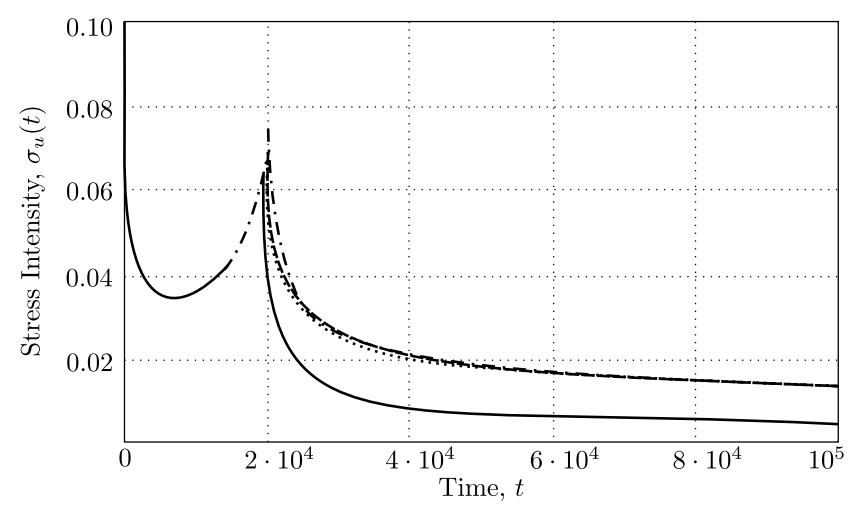

Рис. 7. Зависимости $\sigma_{u}(t)$ при $q=2.5 \cdot 10^{-4}$

[Figure 7. The stress intensity at $q=2.5 \cdot 10^{-4}$ : ideal sliding (solid line), adhesion (dash-dotted line), calculation taking into account friction with the friction coefficient $\mu=0.1$ (dotted line) and $\mu=0.3$ (dashed line)]

ных значениях $q$ к длительностям практически полного заполнения матрицы при этих значениях $q$ :

$$
S=\frac{\tau_{1}}{\tau_{1}^{0}}+\frac{t^{*}-\tau_{2}}{\tau_{2}^{0}}, \quad S=1
$$

В случае идеального скольжения имеем

$$
q^{(1)}=0.5 \cdot 10^{-4}, \quad q^{(2)}=2.5 \cdot 10^{-4}, \quad S=\frac{1}{2}+\frac{t^{* 1}-0.5 \tau_{1}^{0}\left(q^{1}\right)}{\tau_{2}^{0}\left(q^{2}\right)}=0.998<1
$$




$$
q^{(1)}=2.5 \cdot 10^{-4}, \quad q^{(2)}=0.5 \cdot 10^{-4}, \quad S=\frac{1}{2}+\frac{t^{* 2}-0.5 \tau_{1}^{0}\left(q^{2}\right)}{\tau_{2}^{0}\left(q^{1}\right)}=1.00002>1 .
$$

В случае прилипания имеем

$$
\begin{aligned}
& q^{(1)}=0.5 \cdot 10^{-4}, \quad q^{(2)}=2.5 \cdot 10^{-4}, \quad S=\frac{1}{2}+\frac{\bar{t}^{* 1}-0.5 \tau_{1}^{0}\left(q^{1}\right)}{\tau_{2}^{0}\left(q^{2}\right)}=0.94<1 ; \\
& q^{(1)}=2.5 \cdot 10^{-4}, \quad q^{(2)}=0.5 \cdot 10^{-4}, \quad S=\frac{1}{2}+\frac{t^{* 2}-0.5 \tau_{1}^{0}\left(q^{2}\right)}{\tau_{2}^{0}\left(q^{1}\right)}=1.00005>1 .
\end{aligned}
$$

Вычисления показывают, что как в случае идеального скольжения, так и в случае прилипания сумма парциальных времен $S<1$ при мгновенном увеличении величины $q$ и $S>1$ при мгновенном уменьшении величины $q$.

\section{8. Длительная прочность при ступенчатом одноосном нагружении}

В качестве косвенного подтверждения достоверности полученных теоретических результатов рассмотрим результаты известных испытаний металлов на длительную прочность при однократном ступенчатом изменении величины растягивающего напряжения.

Для анализа результатов испытаний при переменной температуре Робинсон [9] предложил правило линейного суммирования парциальных времен. В данном параграфе рассматривается случай, когда растягивающее напряжение в образце равно $\sigma_{1}$ в течение времени $\tau_{1}$, а затем оно скачком меняется до $\sigma_{2}$ и остается таким (без изменения) вплоть до разрушения в момент времени $\tau^{*}=\tau_{1}+\tau_{2}$. Правило суммирования парциальных времен для переменных напряжений часто называется правилом Бейли; для этого случая имеет место следующее равенство: сумма парциальных времен

$$
S=\frac{\tau_{1}}{\tau_{1}^{*}}+\frac{\tau_{2}}{\tau_{2}^{*}}
$$

равна единице $(S=1)$. Здесь под $\tau_{i}^{*}$ понимается время до разрушения при постоянном растягивающем напряжении $\sigma_{i}, i=1,2$.

Многие исследования в той или иной мере подтверждают это правило, однако в значительном количестве работ наблюдаются систематические отклонения от него, выходящие за границы естественного разброса экспериментальных данных. В табл. 5 приведены значения сумм $S$ при возрастании

\section{Таблица 5}

Значения суммы парциальных времен, характеризующие длительную прочность металлов при ступенчатом изменении напряжения [The sum of partial times characterizing the long-term strength of metals with stepwise stress changes]

\begin{tabular}{|c|c|c|c|c|}
\hline \multirow{2}{*}{ Data description } & \multirow{2}{*}{ Material } & Tempera- & \multicolumn{2}{|c|}{$S$} \\
\cline { 4 - 5 } & & ture, ${ }^{\circ} \mathrm{C}$ & $\sigma_{1}<\sigma_{2}$ & $\sigma_{1}>\sigma_{2}$ \\
\hline by V. N. Gulyaev [10] & EI-695R steel & - & $0.77<1$ & $3.15>1$ \\
by D. L. Marriott [11] & Aluminium alloy & 180 & $0.71<1$ & $1.26>1$ \\
by V. V. Osasyuk [12] & EI-826 alloy & 800 & $0.84<1$ & $1.04>1$ \\
\hline
\end{tabular}


и уменьшении растягивающего напряжения [10-12]. Во всех приведенных испытаниях металлов на длительную прочность при кусочно-постоянном растягивающем напряжении сумма $S$ больше 1 при $\sigma_{1}>\sigma_{2}$ и принимает значения между 0 и 1 при $\sigma_{1}<\sigma_{2}$. Этот экспериментальный результат аналогичен полученным в п. 6 теоретическим результатам для рассмотренной конструкции.

\section{Выводы}

Приведено исследование ползучести длинной прямоугольной мембраны в стесненных условиях (внутри жесткой матрицы) под действием переменного поперечного давления. В качестве примера исследуется ползучесть мембраны при однократном мгновенном изменении величины поперечного давления. Рассматриваются три варианта условий на контакте мембраны и матрицы: идеальное скольжение, полное прилипание и трение по закону Кулона. В работе рассматриваются четыре последовательные стадии деформирования мембраны: мгновенное упругое деформирование, ползучесть в свободных условиях, ползучесть мембраны при контакте с боковыми сторонами матрицы и ползучесть мембраны при контакте со всеми сторонами матрицы. Анализ проводится до времени практически полного прилегания мембраны к пространству внутри матрицы. Получены зависимости толщины различных частей мембраны и интенсивности напряжений в мембране от времени. При анализе ползучести в случае кусочно-постоянной зависимости величины давления от времени рассмотрены отличия от правила суммирования парциальных времен заполнения матрицы.

Конкурирующие интересы. Заявляем, что в отношении авторства и публикации этой статьи конфликта интересов не имеем.

Авторский вклад и ответственность. Все авторы принимали участие в разработке концепции статьи и в написании рукописи. Авторы несут ответственность за предоставление окончательной рукописи в печать. Окончательная версия рукописи была одобрена всеми авторами.

Финансирование. Работа выполнена при частичной поддержке Российского научного фонда (РНФ 19-19-00062, Самарский государственный технический университет).

\section{Библиографический список}

1. Odqvist F. K. G. Mathematical Theory of Creep and Creep Rupture. Oxford: Clarendon Press, 1966. ix +168 pp.

2. Качанов Л. М. Основы механики разрушения. М.: Наука, 1974. 312 с.

3. Малинин Н. Н. Ползучесть в обработке металлов. М.: Машиностроение, 1986. 216 с.

4. Локощенко А. М. Ползучесть и длительная прочность металлов. Физматлит: М., 2016. 504 c.

5. Локощенко А. М., Терауд В. В. Ползучесть длинной узкой мембраны в стесненных условиях вплоть до разрушения // ПМТФ, 2013. Т. 54, № 3. С. 126-133.

6. Демин В. А., Локощенко А. М., Жеребцов А. А. Ползучесть длинной прямоугольной мембраны внутри криволинейной матрицы // Изв. вузов. Машиностроение, 1998. № 4-6. C. 41-46.

7. Шестериков С. А., Юмашева М. А. Конкретизация уравнения состояния в теории ползучести // Изв. АН СССР. МTТ, 1984. №1. С. 86-91. 
8. Локощенко А. М., Абросимова Е. А. Установившаяся ползучесть длинной мембраны внутри жесткой матрицы при кусочно-постоянной зависимости скорости изменения поперечного давления от времени // ПМТФ, 2019. Т. 60, № 1. C. 103-113. https://doi . org/10.15372/PMTF20190112.

9. Robinson E. L. Effect of temperature variation on the long time rupture strength of steels // Trans. Am. Soc. Mech. Engrs, 1952. vol. 74, no. 5. pp. 777-780.

10. Гуляев В. Н., Колесниченко М. Г. К оценке долговечности в процессе ползучести при ступенчатом изменении нагрузки // Завод. лаб., 1963. №6. С. 748-752.

11. Marriott D. L., Penny R. K. Strain accumulation and rupture during creep under variable uniaxial tensile loading1// J. Strain Analysis, 1973. vol. 8, no. 3. pp. 151-159. https://doi . org/10.1243/03093247V083151.

12. Осасюк В. В., Олисов А. Н. К вопросу о гипотезах суммирования относительных долговечностей // Пробл. прочн., 1979. №11. С. 31-33.

Математический институт им. В.А. Стеклова Российской академии наук приступает к работе в рамках Государственного контракта № 13.597.11.0043 по теме «Создание электронного архива выпусков научных журналов по тематическому направлению «Математика, физика, информационные технологии». Архив будет размещен на Общероссийском портале Math-Net.Ru.

Предполагается пополнить коллекцию Math-Net.Ru архивами ряда ведущих журналов по математике, физике и информационным технологиям, а также материалами научных мероприятий.

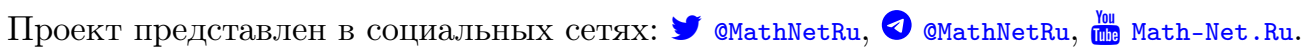




\title{
MSC: 74A05, 74D10
}

\section{The steady-state creep of long membrane in a rigid matrix at a variable transverse pressure}

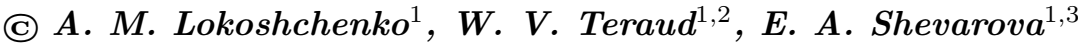 \\ 1 Lomonosov Moscow State University, \\ Institute of Mechanics, \\ 1, Michurinsky prospekt, Moscow, 119192, Russian Federation. \\ 2 Samara State Technical University, \\ 244, Molodogvardeyskaya st., Samara, 443100, Russian Federation. \\ 3 Moscow Aviation Institute (National Research University), \\ 4, Volokolamskoe Shosse, Moscow, 125993, Russian Federation.
}

\begin{abstract}
The problem of the steady-state creep of a long rectangular membrane in constrained conditions inside a rigid matrix is investigated with a piecewise constant dependence of the transverse pressure $q$ on time $t$. The problem considers a long matrix of rectangular cross section, in which the ratio of its height to width is not less than 0.5. As an example, the creep of the membrane is investigated with a single change in the magnitude of the transverse pressure over time. Three variants of the contact conditions of the membrane and the matrix are considered: perfect sliding, adhesion and sliding taking friction into account. In this paper, four stages of membrane deformation were investigated. At the first stage (elastic deformation), the membrane, flat in the initial state, under the action of pressure, instantaneously is deformed elastically, acquiring the form of an open circular cylindrical shell with a central angle $2 \alpha_{1}$. At the second stage, the membrane is deformed under steady-state creep conditions up to the moment when the side walls of the matrix touch. The third stage ends when the membrane touches the transverse wall of the matrix. In the fourth stage, the membrane is in contact with the matrix on the transverse and lateral sides. The analysis is carried out until the time of almost complete adherence of the membrane to the matrix, at which the ratio of the radius of the membrane near the corners
\end{abstract}

\section{Research Article}

(ㅇ)(i) The content is published under the terms of the Creative Commons Attribution 4.0 International License (http://creativecommons.org/licenses/by/4.0/)

Please cite this paper in press as:

Lokoshchenko A. V., Teraud W. V., Shevarova E. A. The steady-state creep of long membrane in a rigid matrix at a variable transverse pressure, Vestn. Samar. Gos. Tekhn. Univ., Ser. Fiz.-Mat. Nauki [J. Samara State Tech. Univ., Ser. Phys. Math. Sci.], 2020, vol. 24, no. 3, pp. 445-468. https://doi.org/10.14498/vsgtu1772 (In Russian).

\section{Authors' Details:}

Alexander M. Lokoshchenko (10) https://orcid.org/0000-0002-5462-6055

Dr. Phys. \& Math. Sci., Professor; Head of Laboratory; Lab. of Creep and Long-Term Strength ${ }^{1}$; e-mail: loko@imec.msu.ru

Walentin V. Teraud (D) https://orcid.org/0000-0001-8336-5598

Cand. Techn. Sci.; Senior Researcher; Lab. of Creep and Long-Term Strength ${ }^{1}$; Researcher; Dept. of Applied Mathematics and Computer Science ${ }^{2}$;

e-mail: 1drnww@gmail .com

Ekaterina A. Shevarova; Lead Engineer; Lab. of Creep and Long-Term Strength ${ }^{1}$; Student $^{3}$; e-mail: kat121193@ya.ru 
of the matrix to the initial width of the membrane is 0.005 . For the third and fourth stages, the friction force of the membrane on the matrix walls is additionally taken into account. The dependences of the thickness of various parts of the membrane on time and on the intensity of stresses in the membrane on time are obtained. In relation to this formulation of the problem, deviations from the rule of summing the partial times of filling the matrix are considered.

Keywords: membrane, steady-state creep, the matrix, transverse pressure, ideal sliding, adhesion, non-stationary loading, friction.

Received: $11^{\text {th }}$ February, $2020 /$ Revised: $10^{\text {th }}$ July, 2020 /

Accepted: $14^{\text {th }}$ September, $2020 /$ First online: 30 ${ }^{\text {th }}$ September, 2020

Competing interests. We declare that we have no conflicts of interest in the authorship and publication of this article.

Authors' contributions and responsibilities. Each author has participated in the article concept development and in the manuscript writing. The authors are absolutely responsible for submitting the final manuscript in print. Each author has approved the final version of manuscript.

Funding. This study was partially supported by the Russian Science Foundation (RSF 1919-00062, Samara State Technical University).

\section{References}

1. Odqvist F. K. G. Mathematical Theory of Creep and Creep Rupture. Oxford, Clarendon Press, 1966, ix +168 pp.

2. Kachanov L. M. Osnovy mekhaniki razrusheniia [Fundamentals of Fracture Mechanics]. Moscow, Nauka, 1974, 312 pp. (In Russian)

3. Malinin N. N. Polzuchest' v obrabotke metallov [Creep in Metal Forming]. Moscow, Mashinostroenie, 1986, 216 pp. (In Russian)

4. Lokoshchenko A. M. Creep and Long-Term Strength of Metals. Boca, Raton, 2018, xviii+545 pp. https://doi.org/10.1201/b22242.

5. Lokoshchenko A. M., Teraud V. V. Creep of a long narrow membrane up to fracture under constrained conditions, J. Appl. Mech. Tech. Phys., 2013, vol.54, no. 3, pp. 451-457. https://doi.org/10.1134/S0021894413030140.

6. Demin V. A., Lokoshchenko A. M., Zherebtsov A. A. Creep of a long rectangular membrane in a curvilinear matrix, Izv. Vuzov. Mashinostroenie, 1998, no. 4-6, pp. 41-46 (In Russian).

7. Shesterikov S. A., Yumasheva M. A. Concretization of the equation of state in the creep theory, Izv. AN SSSR. MTT [Mech. Solids], 1984, no. 1, pp. 86-91 (In Russian).

8. Lokoshchenko A. M., Abrosimova E. A. Steady-state creep of a long membrane in a rigid matrix with a piecewise constant dependence of the rate of change in transverse pressure on time, J. Appl. Mech. Tech. Phys., 2019, vol.60, no.1, pp. 87-96. https://doi.org/ 10.1134/S0021894419010127.

9. Robinson E. L. Effect of temperature variation on the long time rupture strength of steels, Trans. Am. Soc. Mech. Engrs, 1952, vol. 74, no. 5, pp. 777-780.

10. Gulyaev V. N., Kolesnichenko M. G. On the evaluation of service life during the creep process with the load applied in stages, Industrial Laboratory, 1963, vol. 29, no. 6, pp. 799-802.

11. Marriott D. L., Penny R. K. Strain accumulation and rupture during creep under variable uniaxial tensile loading1, J. Strain Analysis, 1973, vol. 8, no. 3, pp. 151-159. https://doi. org/10.1243/03093247V083151.

12. Osasyuk V. V., Olisov A. N. Hypotheses for summing relative endurances, Strength Mater., 1979, vol.11, no.11, pp. 1229-1232. https://doi.org/10.1007/BF00767046. 